Veronica Cimolin*, Paolo Capodaglio, Nicola Cau, Manuela Galli, Cristina Santovito, Alessandra Patrizi, Gabriella Tringali and Alessandro Sartorio

\title{
Computation of spatio-temporal parameters in level walking using a single inertial system in lean and obese adolescents
}

DOI 10.1515/bmt-2015-0180

Received September 15, 2015; accepted October 27, 2016; online first November 29, 2016

Abstract: In recent years, the availability of low-cost equipment capable of recording kinematic data during walking has facilitated the outdoor assessment of gait parameters, thus overcoming the limitations of three-dimensional instrumented gait analysis (3D-GA). The aim of this study is twofold: firstly, to investigate whether a single sensor on the lower trunk could provide valid spatio-temporal parameters in level walking in normal-weight and obese adolescents compared to instrumented gait analysis (GA); secondly, to investigate whether the inertial sensor is capable of capturing the spatio-temporal features of obese adolescent gait. These were assessed in 10 obese and 8 non-obese adolescents using both a single inertial sensor on the lower trunk and an optoelectronic system. The parameters obtained were not statistically different in either normal-weight or obese participants between the two methods. Obese adolescents walked with longer stance and double support phase compared to normalweight participants. The results showed that the inertial

*Corresponding author: Veronica Cimolin, Department of Electronics, Information and Bioengineering, Politecnico di Milano, p.zza Leonardo Da Vinci 32, 20133, Milano, Italy, Phone: +39-02 23993359, Fax: +39-02 23993360,

E-mail: veronica.cimolin@polimi.it

Paolo Capodaglio and Cristina Santovito: Istituto Auxologico Italiano, IRCCS, Orthopaedic Rehabilitation Unit and Clinical Lab for Gait Analysis and Posture, Ospedale San Giuseppe, Via Cadorna 90, I-28824, Piancavallo (VB), Italy

Nicola Cau: Department of Electronics, Information and Bioengineering, Politecnico di Milano, p.zza Leonardo Da Vinci 32, 20133, Milano, Italy

Manuela Galli: Department of Electronics, Information and Bioengineering, Politecnico di Milano, p.zza Leonardo Da Vinci 32 20133, Milano, Italy; and IRCCS “San Raffaele Pisana”, Tosinvest Sanità, Roma, Italy

Alessandra Patrizi, Gabriella Tringali and Alessandro Sartorio: Istituto Auxologico Italiano, IRCCS, Division of Auxology and Experimental Laboratory for Auxo-endocrinological Research, Ospedale San Giuseppe, Via Cadorna 90, I-28824, Piancavallo (VB), Italy system is a valid means of evaluating spatio-temporal parameters in obese individuals.

Keywords: gait; inertial sensor; obesity; validation.

\section{Introduction}

Level walking is a basic skill and the study of gait capacity is a key functional assessment in many conditions. Threedimensional instrumented gait analysis (3D-GA) provides comprehensive data on normal and pathological gait which are useful in clinical practice and research because it provides objective information about joint motion (kinematics), time-distance variables (spatio-temporal data), joint moments and powers (kinetics). It is widely acknowledged that 3D-GA provides crucial information for the determination of the level of functional limitation following a condition. Conventionally, the measurement of kinematics and kinetics of the main body segments and joints is performed by means of optoelectronic marker systems and force plates in specially designed gait laboratories. Optoelectronic-based movement analysis is considered accurate [21], but its use in clinical practice may be limited by the availability of specific laboratories, costs and dependency on trained users [2, 31]. Another critical aspect is related to the positioning of markers on specific body landmarks, which may be difficult in subjects with excessive body fat accumulation.

Low-cost tools may contribute to the implementation of out-patient use of quantitative 3D-GA for clinical purposes. The limitations of measurements confined to laboratory settings can be overcome by recording kinematic data during walking from the total body movements measured with wireless inertial and magnetic devices. Small, lightweight sensor units entailing miniaturised accelerometers, gyroscopes and magnetometers have been extensively developed in recent years. Several studies have been carried out on healthy individuals to validate these systems and different algorithms for the computation of parameters $[1,23,32]$. The data obtained have been used as rehabilitation outcome measures in Parkinson's 
disease and other musculoskeletal-neurological disorders $[8,9,13,16,18,28-30]$. A few studies have used multiple sensors, one for each body segment, and a protocol for gait analysis has been recently developed and applied to individuals suffering from cerebral palsy $[6,10,31]$. Multisensor protocols appear to provide useful data, but even information obtained from a single sensor may be beneficial for clinical purposes. A single, easy-to-wear sensor generally positioned on the lower back presents minimum encumbrance for patients in their habitual environments. Using specific algorithms, this simple method is capable of quantifying gait; the inverted pendulum model is used to evaluate step length and the use of appropriate filtering procedures to identify initial/final contact events within the gait cycle $[14,20]$.

However, validating the accuracy of such simple devices in capturing gait outcomes would appear to be mandatory. Studies on healthy $[2,3,11,27]$ and pathological $[4,8,15,17,18]$ adult subjects have revealed that most gait spatio-temporal parameters can be appropriately assessed. Very few studies on inertial sensor applications for the measurement of gait parameters limited to normalweight subjects $[24,25]$ and Duchenne muscular dystrophy [19] have been published for children. No studies on obese individuals have been published to date. From a clinical point of view, a simple and valid method for quantifying gait strategy in these subjects is essential. Traditional gait analysis relies on accurate marker positioning, which has been shown to be difficult in obese individuals [31]. Traditional gait analysis also requires that the patient wear minimal clothing, which has been shown to cause anxiety in obese individuals [31]. However, the use of inertial sensors to measure gait parameters does not require marker placement or minimal clothing, thus overcoming the typical limitations of measurements in laboratory settings.

Thus, the aim of this study is to validate spatio-temporal parameter estimates in level walking with a single sensor on the lower trunk in obese adolescents. The measurements obtained with this device were compared with the corresponding ones obtained by instrumented 3D-GA.

\section{Materials and methods}

\section{Participants}

In this study, obese $\left(\mathrm{BMI}>97^{\text {th }}\right.$ percentile or $>2 \mathrm{SD}$ from the mean for age and sex) and normal-weight (with BMI between the $25^{\text {th }}$ and $75^{\text {th }}$ percentiles) children were recruited and matched for age and height. In particular, we considered 10 obese adolescents (Obese Group; five males and five females; age: $14-18$ years; BMI: $35.45 \pm 4.73 \mathrm{~kg} / \mathrm{m}^{2}$, height: $1.68 \pm 0.06 \mathrm{~m}$; weight: $100.13 \pm 12.37 \mathrm{~kg}$ ) and eight normal-weight adolescents (Control Group; four males and four females; age: 14-18 years; BMI: $18.67 \pm 2.46 \mathrm{~kg} / \mathrm{m}^{2}$, height: $1.65 \pm 0.10 \mathrm{~m}$; weight: $51.37 \pm 11.21 \mathrm{~kg}$ ).

Exclusion criteria for the obese subjects were musculoskeletal, neuromuscular and/or cardiopulmonary conditions other than obesity that would hinder mobility capacity or contraindicate an integrated weight-management program. Obese subjects were recruited from in-patients admitted for an integrated bodyweight reduction program at the Division of Auxology, Istituto Auxologico Italiano, Piancavallo (VB). They were not involved in regular physical activities before hospitalization ( $<1-2 \mathrm{~h} /$ week). None of them suffered from diabetes and hypertension, pain, headaches, balance disorders and/ or any other symptoms hampering the execution of the tests.

Exclusion criteria for the Control Group included a prior history of cardiovascular, neurological or musculoskeletal disorders. They showed normal flexibility and muscle strength and no obvious gait abnormalities.

The study was approved by the Ethics Research Committee of IRCCS Istituto Auxologico Italiano, Piancavallo (VB), Italy. Written informed consent was obtained from the participants' parents.

\section{Equipment}

Spatio-temporal gait parameters were assessed using both an inertial sensor and an optoelectronic system with passive markers according to the standard instrumented 3D-GA.

The single inertial sensor, a validated [2] wireless inertial sensing device (GSensor, BTS Bioengineering S.p.A., Milano, Italy), was attached using a semi-elastic belt at the lower lumbar level (centred on the L4-L5 intervertebral space; Figure 1) of the participants and provides accelerations along three orthogonal axes: antero-posterior, medio-lateral and supero-inferior. Acceleration data were transmitted via Bluetooth to a PC and processed using the dedicated BTS G-STUDIO version 2.6.12.0 software (BTS Bioengineering S.p.A., Milano, Italy). From the acceleration signals, the typical spatiotemporal gait parameters are automatically obtained using the dedicated BTS G-STUDIO version 2.6.12.0 software. In particular, forward acceleration was found to be the most meaningful signal and the parameters were computed on the basis of the literature [12]. For step detection, the onset of the support phase was determined from the forward acceleration signal. Along the line of progression, the basic pattern of pelvic acceleration corresponds to a pattern predicted by an inverted pendulum model. After low-pass filtering the acceleration signal in the walking direction with a cut-off frequency equal to step frequency, the remaining signal showed a basic pattern of acceleration after mid-stance and deceleration after foot contact. The peak forward acceleration preceding the change of sign was chosen as the initial foot contact (Figure 2). For the calculation of walking distance, step length can be estimated based on the amplitude of vertical pelvic displacement and leg length using a simple inverted pendulum model of walking. Changes in vertical position were calculated by double integration of the superior-inferior acceleration signal. After high-pass filtering to correct for integration drift (4-th order zero-lag Butterworth filter at $0.1 \mathrm{~Hz}$ ), the amplitude of changes in vertical position was determined as the difference between the highest and lowest position during a step cycle. Subsequent step lengths were then calculated as (eq. 1) 


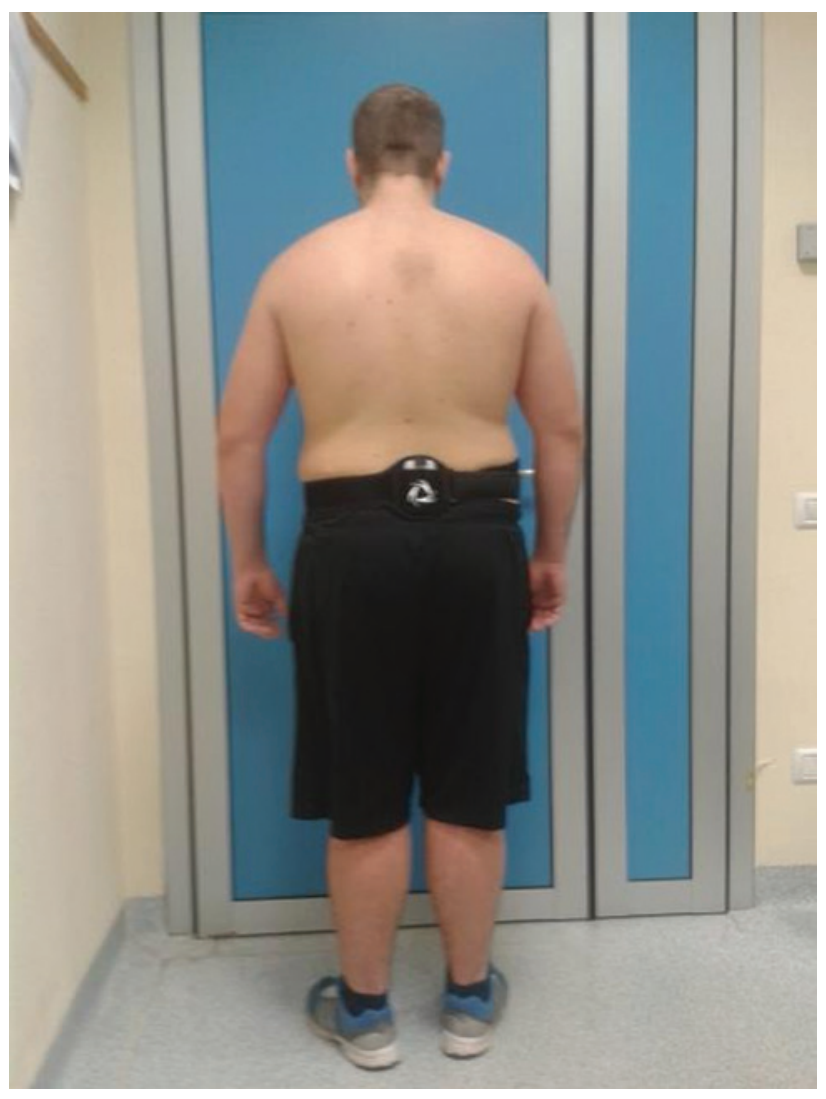

Figure 1: The inertial system used on an obese adolescent.

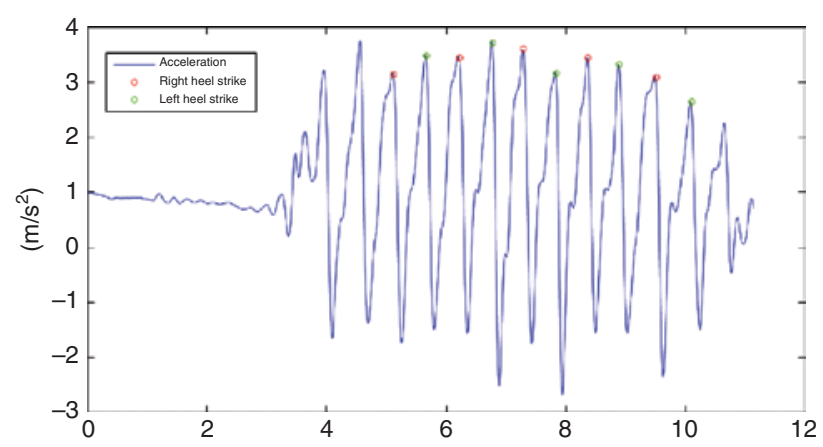

(s)

Figure 2: The change of sign $\left(^{\circ}\right)$ of the peak in the acceleration signal in anterior-posterior direction is taken as the instant of foot contact.

$$
\text { step length }=2 \sqrt{2 \mathrm{lh}-\mathrm{h}^{2}}
$$

with "l" being the individual leg length and ' $h$ ' being the amplitude of vertical displacement during a step cycle. Walking distance (from start to stop of the data collection) was estimated by multiplying mean step length over a certain duration by the number of steps. Velocity was computed as the ratio between walking distance and duration.

From the 3-axial gyroscopic signals, the kinematics of the pelvis in the sagittal, frontal and transversal planes are described [11]. Gyroscopic signals were also acquired during this study, but they are not included in the present analysis and are not discussed in this paper, because the aim was to validate spatio-temporal parameter estimates in level walking with a single sensor on the lower trunk in healthy and obese adolescents.

The system was validated using an optoelectronic system with six cameras (VICON, Oxford Metrics Ltd., Oxford, UK) and two force platforms (Kistler, Einterthur, $\mathrm{CH}$ ). After a number of anthropometric measurements had been collected, passive markers were placed at special points of reference directly on the subject's skin, as described by Davis [7]. Starting from the 3D coordinates of the three markers fixed with each segment (pelvis, thigh, leg and foot), a rigid frame was computed; the Euler angles between two frames are the flex-extension, abdo-adduction and intra-extra rotation of principal joints [7]. Marker position data are also the basis of stride and temporal parameters, such as walking speed, step length, stride length, cadence and so on.

Each subject was fitted with the passive markers and an inertial sensor. The data were thus acquired simultaneously by 3D-GA and inertial sensors. After placement of the markers, the participants completed two or more practice trials across the plate walkway to ensure that they were comfortable with the experimental procedure. After familiarization, the participants were asked to stand up and remain in an upright posture for a few seconds to calibrate sensors and then to walk barefoot at their own natural pace along a 10-m walkway with embedded force platforms at the mid-point. At least five steps for each trial were acquired.

After each track, the participants paused for a few seconds before turning around, paused again and started the new track. This exercise was repeated in order to acquire 10 valid trials for each participant. A trial was considered valid when the following criteria were met: (1) a natural walk at a participant's normal walking pace and (2) a whole foot was measured on the plate. In this way, a total of 100 trials for obese adolescents and 80 trials for non-obese adolescents were collected and analysed.

\section{Data processing}

As previously reported, the first and last steps obtained by the inertial sensor were removed so as to obtain three gait cycles for each side. For 3D-GA data, the first heel strike event for each foot was taken from the force platform, the previous and the following corresponding events from the comparison of that kinematic configuration (foot position, hip/knee/ankle flexion angles, etc.) over the 3D-GA results across the entire data collected. From these events, three full gait cycles for each side were analysed and they were the same as those measured with the inertial sensor.

From the data obtained by the inertial sensor and with 3D-GA, the following spatio-temporal parameters were computed and analysed:

- Stride length (m), the distance between two consecutive heelstrikes of the same foot;

- Stride duration (s), the time between two consecutive heelstrikes of the same foot;

- Stance duration (\%), the foot support phase, i.e. from heel strike to toe off of the same foot, duration as percentage of gait cycle;

- Double support duration (\%), the duration of the phase of support on both feet as percentage of gait cycle;

- Mean velocity (m/s), average instantaneous speed within the gait cycle; from $3 \mathrm{D}-\mathrm{GA}$, the velocity is computed as the ratio between stride length and stride time;

- Cadence (step/min), the number of steps in a minute; 


\section{Statistical analysis}

All the previous parameters were computed for each participant. Kolmogorov-Smirnov tests were used to verify whether parameters were normally distributed; the parameters were not normally distributed and non-parametric analysis was thus used. The median and quartile range values of all the parameters were calculated beforehand for each subject and then for the Control Group and for the Obese Group. The measurements obtained by the inertial system and those obtained by 3D-GA were compared using the Wilcoxon test; obese and non-obese data were compared using the Mann-Whitney U-tests in order to detect significant differences. The research for correlation between 3D-GA and inertial system data in the two groups studied was performed using Spearman's rank-order correlation. In order to evaluate the level of agreement between the two methods, a Bland-Altman plot was performed for both healthy and obese adolescents. The level of significance was set at $p=0.0083(0.05 / 6)$ after a Bonferroni adjustment for multiple comparisons.

\section{Results}

Table 1 shows the results of the spatio-temporal parameters obtained with the inertial sensor and 3D-GA for the normal-weight and obese adolescents.
Firstly, data computed both by 3D-GA and by the inertial sensor were compared, in order to validate the inertial system using instrumented 3D-GA. Data showed that all spatio-temporal parameter values in both normal-weight adolescents and obese participants were not statistically different between the two devices ( $p>0.05$ ).

We then compared normal-weight and obese adolescents' data in terms of 3D-GA and of inertial system parameters. 3D-GA data showed that obese adolescents walked with longer stance and double support phase as compared with normal-weight participants. These results are in line with previous literature on obese adolescents $[5,22]$. The data obtained from the inertial sensors displayed the same results.

The $\mathrm{p}$-values obtained from statistical analysis are displayed in Table 2.

Table 3 shows the results obtained after correlation research in the two groups studied. Both groups showed significant and good correlations for all parameters considered from a statistical viewpoint between data obtained by 3D-GA and the inertial system, respectively $(\mathrm{p}<0.05)$. This means that increases or decreases in data obtained by 3D-GA in general predict the same directional change as the data obtained with the inertial system.

Table 1: Parameters (median and quartile range) obtained from instrumented 3D gait analysis (3D-GA) and the inertial system for the two groups studied.



${ }^{a} \mathrm{p}<0.05$, Obese group vs. control group. No statistical differences were present between GA vs. inertial system data.

Table 2: The p-values obtained by the statistical analysis are shown.

\begin{tabular}{lcrrr}
\hline & \multicolumn{2}{c}{ 3D-GA data vs. inertial system data } & & Control group vs obese group \\
\cline { 2 - 5 } & Control group & Obese group & 3D-GA data & Inertial system data \\
\cline { 3 - 5 } Cadence & 0.1481 & 0.0401 & 0.1094 & 0.1316 \\
Stride duration & 0.1326 & 0.1468 & 0.0908 & 0.1151 \\
Stance duration & 0.1069 & 0.0260 & $\mathbf{1 . 8 6 \mathrm { E } - 0 6}$ & $\mathbf{0 . 0 0 0 5}$ \\
Stride length & 0.0668 & 0.0958 & 0.0182 & 0.0547 \\
Mean velocity & 0.0668 & 0.0554 & 0.1548 & 0.0664 \\
Double support duration & 0.0891 & 0.0318 & $\mathbf{6 . 8 3 E - 0 5}$ & $\mathbf{0 . 0 0 1 5}$ \\
\hline
\end{tabular}

Significant $p$-values, statistically speaking, are shown in bold. The level of significance was set at $p=0.0083(0.05 / 6)$, after a Bonferroni adjustment for multiple comparisons. 
Table 3: Results of correlation research ( $r$-value) between instrumented gait analysis (GA) and inertial system data for the two groups studied.

\begin{tabular}{lrr}
\hline & Control group & Obese group \\
\hline Stride length (m) & $0.75^{\mathrm{a}}$ & $0.70^{\mathrm{a}}$ \\
Stride duration (s) & $0.99^{\mathrm{a}}$ & $0.68^{\mathrm{a}}$ \\
Stance duration (\%) & $0.72^{\mathrm{a}}$ & $0.69^{\mathrm{a}}$ \\
Double support duration (\%) & $0.96^{\mathrm{a}}$ & $0.76^{\mathrm{a}}$ \\
Mean velocity (m/s) & $0.92^{\mathrm{a}}$ & $0.84^{\mathrm{a}}$ \\
Cadence (step/min) & $0.98^{\mathrm{a}}$ & $0.72^{\mathrm{a}}$ \\
\hline
\end{tabular}

${ }^{\mathrm{a}} \mathrm{p}<0.05$

Figures 3 and 4 show the Bland-Altman plots for normal-weight and obese participants. It is a scatter plot of the mean of inertial system and instrumented 3D-GA plotted against the difference between the two methods. This plot provides a visual representation of the level of correlation and in particular, the difference in all the spatio-temporal parameters considered in this analysis determined by both systems. The horizontal line represents the mean of the differences while the dotted lines (computed as the mean of the difference plus or minus 1.96 times its $\mathrm{SD})$ represent the interval of confidence.

The average of the differences allows us to estimate whether one of the two methods underestimated or overestimated the volume measure more than the other. Where the points on the graph are between the two lines the two methods provide consistent results. The Bland-Altman graphic analysis showed good overall correlation between the two systems.

\section{Discussion}

In order to test whether a wireless inertial system is capable of identifying standard gait spatio-temporal parameters during level walking in normal-weight and obese adolescents, the participants' walking abilities were analysed simultaneously with inertial sensors and 3D-GA and the spatio-temporal parameters were compared.

No statistical differences were found between the two systems in terms of all the spatio-temporal parameters analysed. These results confirmed the results of previous studies carried out on healthy adult individuals [2].

In addition, comparison of the parameters between normal-weight and obese individuals both with inertial sensors and instrumented 3D-GA showed that stance phase and double support duration were longer in the Obese Group than in the Control Group. These data suggest that the inertial system is capable of detecting differences between the two groups in accordance with 3D-GA output and in line with the literature [5,22].
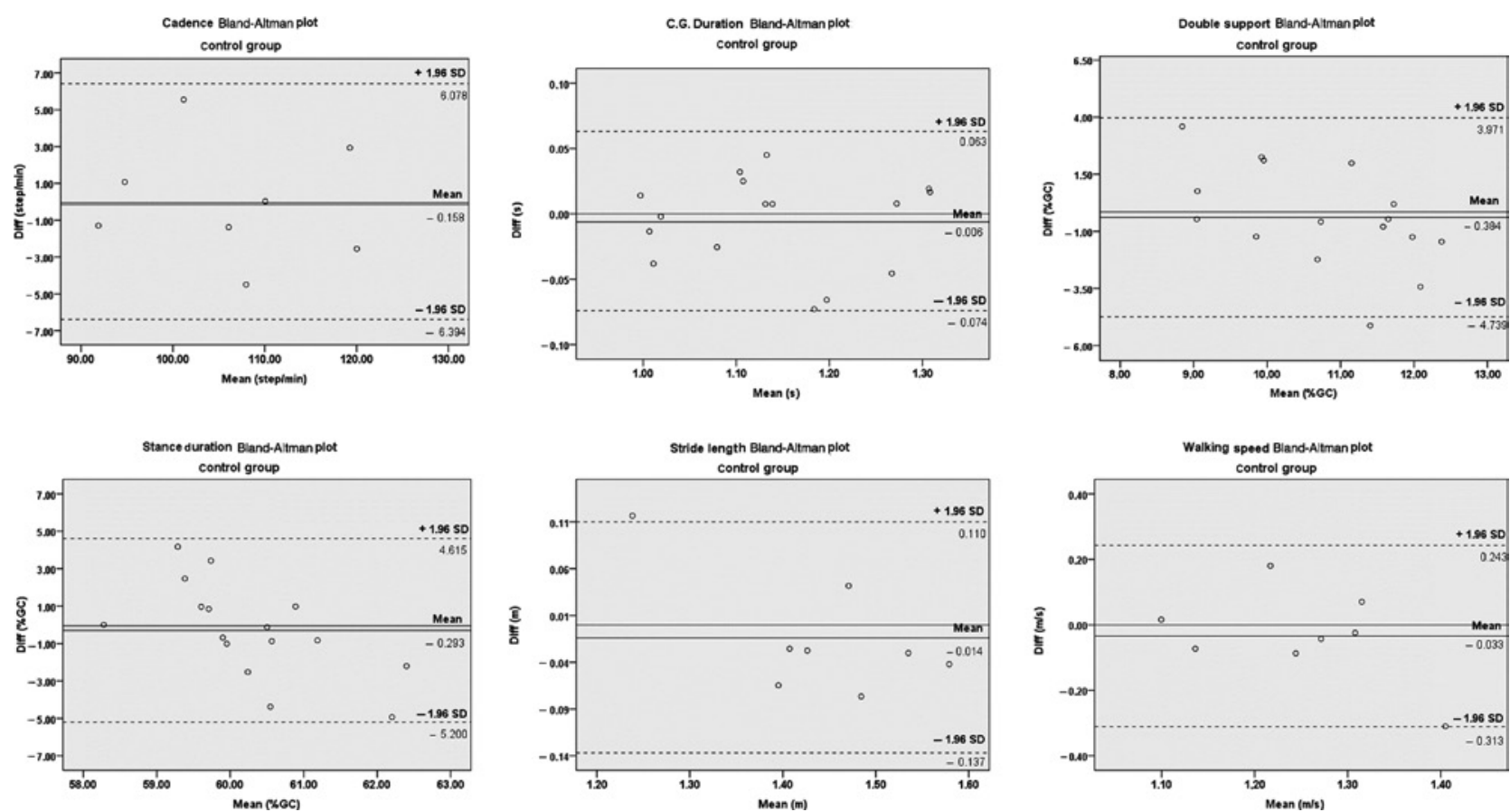

Figure 3: Bland-Altman plots of the mean of instrumented 3D gait analysis (3D-GA) and the inertial system plotted against the difference between the two methods for normal-weight adolescents. 

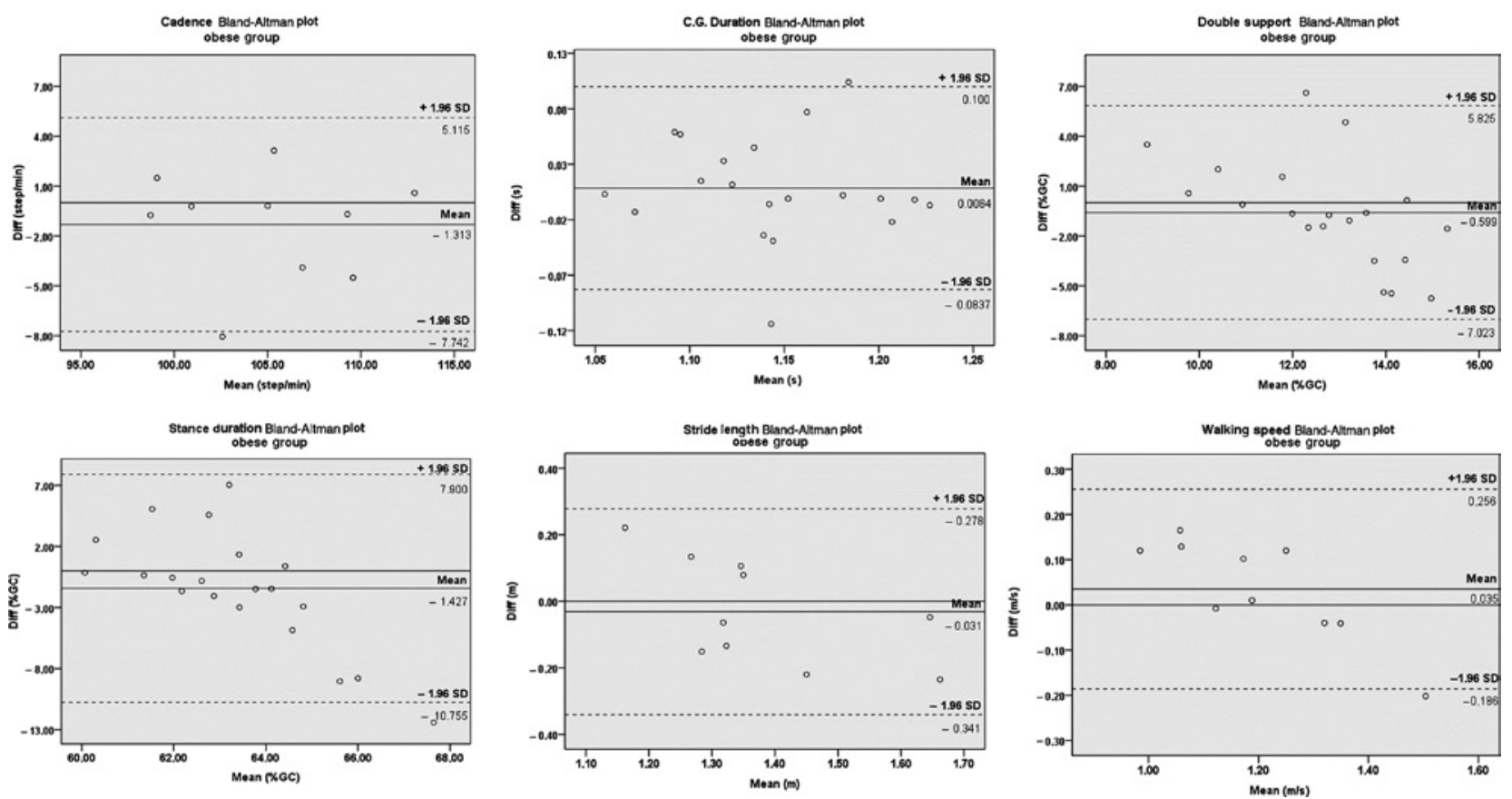

Figure 4: Bland-Altman plots of the mean of instrumented 3D gait analysis (3D-GA) and the inertial system plotted against the difference between the two methods for obese adolescents.

These results show that the inertial system used in the present study is to be considered a good means of evaluating spatio-temporal parameters in obese individuals. These results are very important from a rehabilitation point of view. Monitoring gait parameters using a single sensor placed on the trunk is a very simple testing system, which may be useful in the clinical setting. It allows for in- and outdoor patient evaluation thus overcoming the limitations of gait analysis in laboratory settings and - not of secondary importance - without undressing patients for marker placement, which is frequently a psychological barrier for these individuals. In addition, sensor placement is very easy and quick and, unlike 3D-GA, expert operators are not required.

One of the limitations of this study is that only spatio-temporal parameters were measured although 3D kinematics were available. However, among relevant measurements, spatio-temporal parameters are widely used by clinicians for gait characterisation in obese individuals. They quantify the main gait events and therefore reflect patients' ability to fulfil the general gait requirements, i.e. weight acceptance, single-limb support and swing limb advancement [26]. An asymmetric gait, a prolonged stance or double stance phases and a reduction in the speed of progression are all relevant parameters for the diagnosis of pathological gait and the assessment of functional outcome after treatment. Further 3D pelvis kinematics research, not studied in this paper, should be carried out. The single sensor system can be considered to be a valid means for a preliminary, quick, easy and lowcost evaluation of gait variables even outdoors. This may be useful in clinical settings thus overcoming the limitations of gait analysis in laboratory settings.

For a more in-depth assessment of gait strategy in a clinical setting and for the purposes of clinical decisionmaking, this system cannot replace gait laboratories based on optoelectronic systems. However, outpatient rehabilitation facilities could definitely benefit from the information obtained by a single sensor, which provides low-cost quantitative gait parameters, which interfere minimally with a subject's habitual tasks outside the laboratory too.

A possible bias of the study is its relatively small sample size, which limits the validity of its clinical and statistical findings. Further studies should be conducted on a larger number of participants, both healthy and obese. With a larger participant sample the effects of different BMIs on inertial sensor outputs might also be able to be studied in depth. In addition, as one of the outputs of inertial sensor is pelvic kinematics, further investigations could be designed to validate the system in terms of pelvic position assessment, which was not investigated in this study.

Acknowledgments: The authors would like to acknowledge Eng. Lea Caramma for her valuable contribution. 


\section{References}

[1] Auvinet B, Berrut G, Touzard C, et al. Reference data for normal subjects obtained with an accelerometric device. Gait Posture 2002; 16: 124-134.

[2] Bugané F, Benedetti MG, Casadio G, et al. Estimation of spatialtemporal gait parameters in level walking based on a single accelerometer: validation on normal subjects by standard gait analysis. Comput Meth Prog Bio 2012; 108: 129-137.

[3] Buganè F, Benedetti MG, D’Angeli V, Leardini A. Estimation of pelvis kinematics in level walking based on a single inertial sensor positioned close to the sacrum: validation on healthy subjects with stereophotogrammetric system. Biomed Eng Online 2014; 13: 146.

[4] Calliess T, Bocklage R, Karkosch R, Marschollek M, Windhagen $\mathrm{H}$, Schulze M. Clinical evaluation of a mobile sensor-based gait analysis method for outcome measurement after knee arthroplasty. Sensors (Basel) 2014; 14: 15953-15964.

[5] Cimolin V, Galli M, Vismara L, Albertini G, Sartorio A, Capodaglio P. Gait pattern in lean and obese adolescents. Int J Rehabil Res 2015; 38: 40-48.

[6] Cutti AG, Ferrari A, Garofalo P, et al. 'Outwalk': a protocol for clinical gait analysis based on inertial and magnetic sensors. Med Biol Eng Comput 2010; 48: 17-25.

[7] Davis RB, Ounpuu S, Tyburski D, Gage JR. A gait analysis data collection and reduction technique. Hum Mov Sci 1991; 10: 575-587.

[8] Del Din S, Godfrey A, Rochester L. Validation of an accelerometer to quantify a comprehensive battery of gait characteristics in healthy older adults and Parkinson's disease: toward clinical and at home use. IEEE J Biomed Health Inform 2015; 99: 1-10.

[9] Esser P, Dawes H, Collett J, Feltham MG, Howells K. Validity and inter-rater reliability of inertial gait measurements in Parkinson's disease: a pilot study. J Neurosci Meth 2012; 205: 177-181.

[10] Ferrari A, Cutti AG, Garofalo P, et al. First in vivo assessment of "Outwalk": a novel protocol for clinical gait analysis based on inertial and magnetic sensors. Med Biol Eng Comput 2010; 48: 1-15.

[11] Floor-Westerdijk MJ, Schepers HM, Veltink PH, van Asseldonk $\mathrm{EH}$, Buurke JH. Use of inertial sensors for ambulatory assessment of center-of-mass displacements during walking. IEEE Trans Biomed Eng 2012; 59: 2080-2084.

[12] Ganea R, Jeannet PY, Paraschiv-Ionescu A, et al. Gait assessment in children with duchenne muscular dystrophy during long-distance walking. J Child Neurol 2012; 27: 30-38.

[13] Godfrey A, Conway R, Meagher D, O'Laighin G. Direct measurement of human movement by accelerometry. Med Eng Phys 2008; 30: 1364-1386.

[14] González RC, López AM, Rodriguez-Uría J, Álvarez D, Alvarez JC. Real-time gait event detection for normal subjects from lower trunk accelerations. Gait Posture 2010; 31: 322-325.

[15] Grimpampi E, Bonnet V, Taviani A, Mazzà C. Estimate of lower trunk angles in pathological gaits using gyroscope data. Gait Posture 2013; 38: 523-257.
[16] Horak F, King L, Mancini M. Role of body-worn movement monitor technology for balance and gait rehabilitation. Phys Ther 2015; 95: 461-470.

[17] Ishigaki N, Kimura T, Usui Y, et al. Analysis of pelvic movement in the elderly during walking using a posture monitoring system equipped with a triaxial accelerometer and a gyroscope. J Biomech 2011; 44: 1788-1792.

[18] Kleiner A, Galli M, Gaglione M, et al. The parkinsonian gait spatiotemporal parameters quantified by a single inertial sensor before and after automated mechanical peripheral stimulation treatment. Parkinsons Dis 2015; 2015: 390512.

[19] Masci I, Vannozzi G, Bergamini E, Pesce C, Getchell N, Cappozzo A. Assessing locomotor skills development in childhood using wearable inertial sensor devices: the running paradigm. Gait Posture 2013; 37: 570-574.

[20] McCamley J, Donati M, Grimpampi E, Mazzà C. An enhanced estimate of initial contact and final contact instants of time using lower trunk inertial sensor data. Gait Posture 2012; 36: 316-318.

[21] McGinley JL, Baker R, Wolfe R, Morris ME. The reliability of three-dimensional kinematic gait measurements: a systematic review. Gait Posture 2009; 29: 360-369.

[22] McGraw B, McClenaghan BA, Williams HG, Dickerson J, Ward DS. Gait and postural stability in obese and nonobese prepubertal boys. Arch Phys Med Rehabil 2000; 81: 484-489.

[23] Menz HB, Lord SR, Fitzpatrick RC. Acceleration patterns of the head and pelvis when walking on level and irregular surface. Gait Posture 2003; 18: 35-46.

[24] Mizuike C, Ohgi S, Morita S. Analysis of stroke patient walking dynamics using a tri-axial accelerometer. Gait Posture 2009; 30: 60-64.

[25] Pau M, Mandaresu S, Leban B, Nussbaum MA. Short-term effects of backpack carriage on plantar pressure and gait in schoolchildren. J Electromyogr Kinesiol 2015; 25: 406-412.

[26] Perry J, Burnfield JM. Phases of gait. In: gait analysis normal and pathological function. Thorofare, NJ, USA: SLACK incorporated 2010: 9-16.

[27] Rigoberto MM, Otniel PR, Juan-Carlos AV, Daniel LE, Arturo MM. Analysis of subtle movements related to neurodegenerative diseases using wearable inertial sensors: a study in healthy subjects. Conf Proc IEEE Eng Med Biol Soc 2013; 2013: 6119-6122.

[28] Rueterbories J, Spaich EG, Larsen B, Andersen OK. Methods for gait event detection and analysis in ambulatory systems. Med Eng Phys 2010; 32: 545-552.

[29] Salarian A, Russmann H, Vingerhoets FJG, et al. Gait assessment in Parkinson's disease: toward an ambulatory system for long-term monitoring. IEEE T Biomed Eng 2004; 51: 1434-1443.

[30] Schwesig R, Leuchte S, Fischer D, Ullmann R, Kluttig A. Inertial sensor based reference gait data for healthy subjects. Gait Posture 2011; 33: 673-678.

[31] van den Noort JC, Ferrari A, Cutti AG, Becher JG, Harlaar J. Gait analysis in children with cerebral palsy via inertial and magnetic sensors. Med Biol Eng Comput 2013; 51: 377-386.

[32] Zijlstra W, Hof AL. Assessment of spatio-temporal gait parameters from trunk accelerations during human walking. Gait Posture 2003; 18: 1-10. 